\title{
iPSC for Dental Tissue Regeneration
}

\author{
Kim Hynes • Stan Gronthos • P. Mark Bartold
}

Published online: 18 January 2014

(C) Springer International Publishing AG 2014

\begin{abstract}
The realisation that adult somatic cells can be reprogrammed into pluripotent cells is revolutionising the way diseases are researched and is set to transform the way diseases are treated. In recent years the use of induced pluripotent stem cells (iPSC) in dentistry has begun to be investigated. Whilst this work is still in its infancy, iPSC are demonstrating great potential for use in the regeneration of dental tissues. In this review we will provide a brief introduction to the properties of iPSC and their potential application as therapeutic agents to enhance medical research. Furthermore, this review will summarise recent developments in the use of iPSC in dental tissue regeneration.
\end{abstract}

Keywords iPSC $\cdot$ Dental tissue regeneration $\cdot$ Stem-cell biology $\cdot$ Diseases $\cdot$ Adult somatic cells

\section{Introduction}

Induced pluripotent stem cells (iPSC) were first generated through groundbreaking work performed by Yamanaka and colleagues in 2006. Their work revealed that somatic or terminally differentiated cells could be reprogrammed back into a pluripotent state through forced expression of a cocktail of transcription factors [1]. In 1962 John Gurdon first discovered that specialisation of cells was reversible; however, it took a further 44 years to identify the specific factors required for the initiation of this process. The revolutionary extent of Yamanaka's and Gurdon's work was acknowledged by the Noble prize award in Physiology or Medicine in 2012. It has been suggested that iPSC technology will accelerate our

\footnotetext{
K. Hynes $(\bowtie) \cdot$ P. M. Bartold

School of Dentistry, Colgate Australian Clinical Dental Research

Centre, University of Adelaide, Adelaide, SA, Australia

e-mail: kim.hynes@adelaide.edu.au

S. Gronthos

Mesenchymal Stem Cell Laboratory, School of Medical Sciences, University of Adelaide, Adelaide, SA, Australia
}

understanding of human diseases by developing models of genetic disorders, as a tool for studying developmental biology, drug discovery and the potential for genetic engineering utilising stem cell based regenerative therapies.

\section{The Development of iPSC}

iPSC were first generated from mouse embryonic fibroblasts and adult tail tip fibroblasts through forced expression of four transcription factors: Oct3/4, Sox2, c-Myc and Klf4 [1]. Independent research performed by Thomson and colleagues ascertained a different cocktail of transcription factors, OCT4, SOX2, NANOG and LIN28, which were capable of reprogramming human foreskin fibroblast and human lung cells to a pluripotent state [2]. Pluripotency is defined by the ability of a cell to give rise to all lineages of the mature organism under the correct stimulus.

The discovery of iPSC has been heralded as having the potential to revolutionise human medicine. The high proliferative capacity and the ability of iPSC to differentiate into all tissue types in the body has introduced the possibility of "persionalised medicine", whereby a patient's own cells could be used to provide biologically compatible cells or tissues for tailored treatments. Whilst the use of patient-specific iPSC would be advantageous to avoid possible rejection in tissue regeneration procedures, the high cost and the lengthy time frame involved in generating iPSC from an individual patient mean that patient-specific iPSC are unlikely to be feasible on a wide scale. However, Nakatasuji et al. have estimated that as few as 50 unique iPSC lines, which are homozygous for the three HLA loci, will be sufficient to provide a perfect HLA match to $90 \%$ of the Japanese population [3, 4]. Therefore it is more realistic to think that iPSC banks will be used to treat patients with HLA-matched donor iPSC rather than iPSC generated from their own tissues.

Whilst iPSC hold vast potential to revolutionise human medicine, major concerns need to be addressed before they can be considered for use in mainstream treatment approaches. 
Their genomic instability, high proliferative capacity and pluripotency support tumour formation in vivo and as such present as fundamental limiting factors in iPSC utilisation. Numerous strategies are being investigated to minimise the carcinogenic properties of iPSC.

One area where advances have been made to minimise the tumorigenicity of iPSC is in the method of transcription factor delivery. In the original protocols used to generate iPSC, integrating viruses were used $[1,2]$. These integrating viruses permanently integrated the exogenous transcription factors into the gemone $[1,2]$ and this integration has been associated with tumour development. Researchers have successfully used nonviral and non-integrating approaches such as non-viral minicircle vectors [5], small molecules [6], excisable lentiviral cassette [7, 8], protein [9], microRNA [10, 11] and synthetic modified mRNA [12] to generate iPSC. Whilst these approaches are considered safer than the traditional protocols for generating iPSC, issues still remain with regard to the presence of residual undifferentiated iPSC from cultures as they can uncontrollably proliferate and form teratomas in vivo.

In terms of regenerative therapies involving iPSC, it is most likely that the iPSC will be differentiated into lineage-specific progenitor cells or mature cell populations prior to implantation into patients in order to form the required functional tissue in vivo. There is however major concern in terms of cell therapies with regard to the presence of residual undifferentiated or partially differentiated cells that could give rise to tumours. Strategies have been suggested and are being investigated to address this issue. These strategies involve the use of suicide genes or chemotherapy to remove teratomas or any cells that go rogue after implantation [13, 14]. Alternatively cell-sorting methods to remove undifferentiated or teratomaforming cells using specific antibodies are being investigated $[15,16]$.

The tumorigenic potential of iPSC or cells derived from iPSC remains a genuine concern for any potential use of iPSC in regenerative therapies; however, the growing number of positive results obtained from iPSC-based cell therapy in preclinical animal models displays the vast potential of these cells and demonstrates the importance of continuing to interrogate and address these safety issues.

\section{Potential of iPSC to Aid Medical Research}

The two main areas where iPSC are set to aid medical research are identified as first a novel research tool for modelling diseases and second potential new treatment approaches.

iPSC Based Research Models

It is becoming clear that iPSC are emerging as an invaluable tool for understanding the processes and pathways involved in diseases. Traditionally animal models (in particular rodent models) have been used to study human diseases; however these models do not always replicate the phenotype displayed in humans. A lack of adequate models of diseased and normal tissue function has limited our understanding of human development and disease pathogenesis.

iPSC technology allows disease-specific stem cells to be produced from affected individuals and healthy relatives. Furthermore disease-specific stem cells can, in theory, be differentiated into the cell types affected by the disease, allowing for enhanced interrogation of a genetic defect or disease on the relevant cell type. It is hoped that by better understanding the processes and pathways involved in a disease scientists will improve their ability to generate novel or improved therapies to treat the disease.

Numerous disease-specific iPSC lines have been generated successfully, including but not limited to diseases affecting the neuronal system [17], immune system [17, 18], blood [19, 20], heart [21, 22] and pancreas [17, 23]. More specifically iPSC-based disease models with robust in vitro disease phenotypes have been generated from a range of monogenic diseases including, but not limited to, spinal muscular atrophy (SMA) [24], long QT syndrome $[21,22]$, various models of liver disease (such as familial hypercholesterolaemia, glycogen storage disease type $1 \mathrm{~A}$ and $\alpha 1$-anti-trypsin deficiency [25]) as well as various neurological disorders (including fragile $\mathrm{X}$ syndrome [26], Rett syndrome [27, 28], Dravet syndrome [29], schizophrenia [30] and Parkinson's disease [31]). Whilst the use of disease-specific iPSC is still in its infancy, positive results have already been generated. One of the early examples of the usefulness of disease-specific iPSC was derived from patients with familial dysautonomia (FD) [32]. FD is a progressive neurodegenerative disorder caused by mutations in the IKBKAP gene. iPSC generated from FD patients express multiple disease-specific phenotypes including decreased expression of IKBKAP in FDiPSC-derived neural crest cells [32]. Furthermore, neuronal differentiation of FD-iPSC identified defects in neurogenesis and migration in neural crest-derived cells [32]. These results revealed new insights into the disease pathogenesis of FD and expose new mechanisms that could be targeted in future therapies.

\section{The Use of iPSC to Treat Diseases}

In addition to the use of iPSC as research models, iPSC also have vast potential for use in the treatment of diseases. Three main applications of iPSC are being investigated with regard to their use in diseases treatment; these are to aid in the discovery of novel therapeutic drugs, genetic correction of mutant genes and as stem cell populations for tissue regeneration. 


\section{Utilisation of iPSC for Drug Discovery Screens}

Disease-specific iPSC that display a measurable disease phenotype in vitro present significant potential to be utilised as a cell source for large-scale drug discovery screens. Recently, Lee et al. have successfully utilised disease-specific FD-iPSC to identify candidate drugs that could have therapeutic potential in affected patients [33]. The authors utilised neural crest precursors derived from iPSC from FD patients to screen 6,912 small molecules for their ability to rescue the expression of IKBKAP in these cells [33]. Their screen identified eight molecules that had the ability to increase the expression of $I K B K A P$; these molecules are now being assessed for their suitability for clinical use [33]. This study demonstrates the feasibility of using iPSC in drug discovery.

\section{Genetic Correction}

iPSC have been hailed as a potential cure for genetic and degenerative diseases through genetic correction, whereby, defective genes can be replaced in disease-specific iPSC as a treatment approach. The potential of this approach was first demonstrated in a proof of principal study in a mouse model of sickle cell anaemia [34]. Hanna et al. demonstrated that implantation of genetically corrected iPSC-derived haematopoietic progenitor cells improved the haematological and systemic parameters of sickle cell anaemia-affected mice to levels comparable with control (non-diseased) mice [34]. Alternatively, genetic correction can be performed on somatic cells prior to being reprogramed to iPSC, as has been demonstrated in Fanconi anaemia (FA) [35]. In this study somatic cells isolated from patients with FA underwent genetic correction before being used to generate iPSC [35]. The resulting corrected Fanconianaemia-specific iPSC were found to be indistinguishable from ES and iPSC from healthy individuals. Importantly the corrected Fanconi-anaemia-specific iPSC gave rise to phenotypically normal and disease-free haematopoietic progenitors [35].

\section{Cell Regeneration}

It is proposed that iPSC have the capacity to treat diseases involving the loss of cellular function or cellular deficiency; hence iPSC could be used to generate replacement cells for implantation into affected patients. Due to the issues regarding the tumorigenicity of iPSC and their derivatives, which have been discussed above, this area of iPSC research is not as advanced as others. The effectiveness of iPSC in cellular regeneration in humans will rely on efficient differentiation of iPSC into a required cell type, cell purification to remove partially differentiated and potentially tumorigenic cells, and finally ascertaining effective delivery methods and/or appropriate scaffolds for delivering the cells to the required site. Whilst the efficacy and safety of iPSC therapies have yet to be demonstrated, a recent study has reported the use of iPSC to aid bone regeneration in critical-sized calvarial defects in rats [36]. In this study, iPSC were cultured on polymeric nanofibrous polyethersulfone (PES) to support the proliferation and osteogenic differentiation of the iPSC in vitro. Subsequent implantation of the iPSC and PES scaffold into calvarial bone defects resulted in enhanced bone regeneration [36]. This study demonstrates the potential held by iPSC in aiding bone regeneration; however, the issue of un-differentiated PSC is not addressed and would be of concern in translating this work to human patients.

With the few examples discussed here it is evident that iPSC have a vast potential for aiding current protocols in human disease treatment and research.

\section{iPSC and Dentistry}

Numerous dental tissues have successfully been used to generate iPSCs, including; human exfoliated deciduous teeth [37•], dental pulp stem cells [37•], stem cells from apical papilla [37•], oral mucosa [38], third molar mesenchymal stromal cells [39], dental pulp [40], periodontal ligament fibroblasts [41, 42], gingival fibroblasts [41, 43] and immature dental pulp stem cells [44]. A comparative study conducted by Yan et al. (2010) reported that iPSC could be generated more efficiently from dental-derived mesenchymal-like stem cells than other somatic cells such as foreskin fibroblasts, skin fibroblasts and adult MSCs [37॰]. Dental-derived stem cells are an attractive source of somatic cells for iPSC generation as their multipotency, high proliferation rates and relative accessibility make them ideally suited for iPSC generation. Furthermore dental pulp cells (DPC) have been identified as an ideal cell source for generating iPSC for cell banking [40] as DPC can easily be obtained from third molar teeth (wisdom teeth), which are routinely extracted from patients.

As greater insight is gained in the capabilities and characteristics of iPSC it is becoming apparent that not all iPSC are equivalent. A number of studies have demonstrated that iPSC retain epigenetic memory of their former tissue type and that this memory can affect the differentiation potential of iPSC $[45,46]$. The epigenetic memory of iPSC is believed to result in iPSC preferentially differentiating back into their tissue of origin. The use of dental-derived iPSC could therefore be advantageous for dental tissue repair.

\section{The Efficacy of iPSC in Dental Tissue Regeneration}

Differentiation of iPSC into Dental Mesenchymal Cells and Tooth Structures

Regenerative dentistry is an emerging field of dental research that investigates the use of stem cells for tissue engineering. 
There are many aspects of dentistry in which the current therapeutic options fail to consistently and reliably treat patients. Tissue engineering is being considered as an alternative option to overcome shortcomings in a range of dental areas including periodontics, endodontics and maxillofacial surgery. In recent years adult stem cells have demonstrated their capacity to regenerate a range of dental structures and even whole teeth [47]. However, the lack of accessibility of adult stem cells is a major roadblock to translating their regenerative potential into novel clinical treatment strategies. This is where iPSC could play a major role in dentistry as they have a limitless expansion capability and would be able to provide the stem cell numbers required for use in tissue regenerative procedures in patients. Whilst there has been a lot of research into the regenerative potential of adult stem cells, the regenerative potential of iPSC as applied to dentistry is a newly emerging field of research, with the first studies being published in the last few years.

The first indication that iPSC had the capacity to differentiate into dental tissues came from Arakaki et al who demonstrated that co-culture of mouse iPSC (miPSC) with dental epithelium lead miPSC to differentiate into ameloblasts [48 $]$. Epithelial-mesenchymal interactions are important interactions in tooth development as they regulate the growth and morphogenesis of teeth. Arakaki et al. investigated the potential of miPSC as a novel source of mesenchymal cells for tooth development. Co-culture of miPSC with epithelial cells resulted in the formation of cells with an epithelial-like morphology that expressed the epithelial cell markers, p63 and cytokeratin-14 as well as the ameloblast markers, ameloblastin and enamelin [48•]. Ameloblasts are an essential cell population required for tooth development as they are involved in enamel formation. These results demonstrated that miPSC have the ability to differentiate into an odontogenic cell fate when co-cultured with dental epithelial cells [48•].

More recently, it was shown that miPSC can differentiate into dental mesenchymal cells (DMC) [49]. Otsu et al. differentiated miPSC into an intermediate population of neural crestlike cells (NCLC) then assessed the potential of the miPSCderived-NCLC for generating dental tissues. NCLC were selected as the intermediate cell type as mesenchymal cells derived from cranial neural crest (NC) cells interact with the dental epithelium during tooth development and this interaction leads to tooth formation $[50,51]$. NC cells are critical for tooth organogenesis as they contribute to the formation of dental mesenchymal cells, which subsequently differentiate into dentin-secreting odontoblasts [52]. When cultured with dental epithelial cells miPSC-derived-NCLC differentiated into cells expressing the DMC markers, Lhx6, Msx1 and Pax6 as well as the odontoblast marker dentin sialoprotein (DSP) [49], thereby demonstrating that miPSC-derived-NCLC had the potential to differentiate into odontoblast progenitor cells under appropriate stimulation. Additionally culture of miPSC-derived-NCLC in serum or conditioned media from dental epithelial cells was sufficient to enhance expression of DSP and dentin morphological protein 1 (DMP1), suggesting that serum and soluble components from dental epithelial cells were sufficient to differentiate the miPSC-derived NCLC into odontoblasts [49]. Conversely, undifferentiated miPSC cultured with dental epithelial cells did not express DSP, demonstrating that differentiation of miPSC to NCLC enhances their ability to differentiate into odontoblasts [49]. This finding highlights the potential application of using miPSC-derived NCLC as a stem cell source for tooth regeneration and tooth development research. Furthermore the miPSC-derived cells were substantially less tumorigenic than undifferentiated miPSC.

This research has been extended in vivo, where the role of miPSC in tooth tissue engineering was investigated [53]. Wen et al. established that miPSC can be induced to express gene expression profiles highly reminiscent of those of osteogenic and odontogenic genes [53]. Furthermore, it was demonstrated that miPSC had the capacity to differentiate in vivo into odontogenic cells in a recombinant tooth germ model [53]. Implantation of miPSC, in combination with epithelial and mesenchymal cells, leads to the formation of bone-like structures, dentin-like and dental pulp-like structures in some instances and in others dental-like structures [53]. Interestingly, implantation of miPSC alone did not form tooth- or bone-like structures. Alternatively implantation of epithelial and mesenchymal cells in the absence of miPSC did form tissueengineered tooth-like structures (TET); however they were irregular. Whilst implantation of miPSC alone failed to form tooth-like structures, when combined with epithelial and mesenchymal cells, they were capable of forming mature TET, which were similar to a normal tooth. Staining revealed the formation of complete new tooth structures that contained miPSC-derived cells. These results demonstrate that miPSCderived cells can differentiate into bone-like and dental-pulplike structures as well as aid tooth development [53]. These results also highlight the importance of cell-cell interactions in tissue engineering as these conditions were essential for the miPSC to display their capacity to enhance tooth regeneration [53].

More recently human iPS cells have been assessed for their capacity to differentiate into dental tissues. Epithelial sheets derived from human iPSC are capable of differentiating into tooth structures when co-cultured with mouse dental mesenchymes [54•]. Implantation of iPSC-derived epithelial sheets with mouse dental mesenchyme cells resulted in the formation of tooth-like structures $30 \%$ of the time. The resulting toothlike structures contained dental pulp, dentin, enamel space and enamel organ. Analysis of the chemical composition and physical properties of the tooth-like structures formed revealed they were similar to regular human teeth [54•]. Assessment of human specific antigen expression demonstrated that only the epithelial components of the tooth had arisen from the 
implanted differentiated iPSC, whilst the dental pulp, cartilage and surrounding bone-like structures originated from the mouse dental mesenchymal cells rather than the iPSC. These findings were expected based on previous research into tooth development where it has been shown that epithelial cells give rise to enamel-forming ameloblasts while MSC form dentinforming odontoblasts and dental pulp. Once again the importance of cell-cell interactions was demonstrated as mouse dental mesenchymal cells alone resulted in the formation of bone-like structures rather than tooth-like structures [54•]. Therefore epithelial sheets derived from iPSC can function as a novel source of epithelial cells for tooth development.

Collectively, these studies demonstrate the potential of iPSC for tooth regeneration. Given that iPSC have been shown to have the capacity to act as a novel source of mesenchymal cells [48•] as well as epithelial sheets [54•] it is likely to only be a matter of time until these two capabilities of iPSC are combined and we see the first creation of an entire tooth from iPSC.

\section{The Use of iPSC in Periodontal Tissue Regeneration}

The use of regenerative dentistry is also being investigated as a novel treatment approach for periodontal disease. In the past this research has typically involved the use of MSC; recently, iPSC have also been investigated for their potential to regenerate periodontal tissues.

The first study looking at iPSC in periodontal regeneration involved the implantation of iPSC into surgically created periodontal fenestration defects in mice [55]. In this study, iPSC in combination with enamel matrix derivatives/ Emdogain gel (EMD $\left.{ }^{\mathrm{TM}}\right)$ were shown to enhance the regeneration of periodontal tissues [55]. EMD ${ }^{\mathrm{TM}}$ has been shown to stimulate growth of multiple MSC types and enhance expression of tissue-specific maturation markers leading to greater regeneration of periodontal tissues [56-60]. Implantation of miPSC combined with EMD ${ }^{\mathrm{TM}}$ into the periodontal defects resulted in significantly greater bone regeneration than in the defects treated with EMD ${ }^{\mathrm{TM}}$ alone [55]. Furthermore, defects that received miPSC and $\mathrm{EMD}^{\mathrm{TM}}$ displayed an almost intact layer of regenerated cementum and new PDL formed between the newly regenerated cementum and alveolar bone, as was not displayed in control defects [55]. This work demonstrates that miPSC have the capacity to regenerate periodontal tissues [55].

Our research group has ameliorated this concept and furthered it by implanting MSC-like cells derived from iPSC into a rat periodontal fenestration defect model [61•]. Implantation of MSC-like cells derived from iPSC into periodontal defects in rats leads to a significant increase in the amount of regeneration and newly formed mineralised tissue present [61•]. MSC-like cells derived from iPSC are emerging as a promising stem cell population for use in regenerative medicine as they incorporate the proliferative advantages of iPSC whilst eliminating the tumorigenicity associated with iPSC [62-66]. Therefore iPSC-MSC-like cells are an easily accessible source of stem cells that could be used in tissue regeneration approaches to periodontitis [61•] and possibly other disorders involving the loss of dental tissues.

\section{Conclusion}

iPSC are emerging as a promising new source of stem cells for use in dental tissue regeneration with proven capacity to regenerate tooth structures and enhance periodontal regeneration. Whilst iPSC have predominantly been investigated for their capacity to regenerate dental tissue there are a wide range of other areas of dentistry where iPSC are likely to have beneficial effects in the years ahead. For example iPSC could be used to model human genetic oral diseases or disorders that involve dental defects; here the generation of disease-specific iPSC will be beneficial for investigating the mechanisms involved in these disorders and enhancing our understanding of dental tissue development. Furthermore, iPSC could be utilised in genetic correction of oral diseases and are also likely to play a role in future drug screens to identify novel drugs for dental therapies.

\section{Compliance with Ethics Guidelines}

Conflict of Interest Dr. Kim Hynes and Dr. Stan Gronthos both declare no potential conflicts of interest relevant to this article.

Dr. P. Mark Bartold serves as a section editor for Current Oral Health Reports.

Human and Animal Rights and Informed Consent This article does not contain any studies with human or animal subjects performed by any of the authors.

\section{References}

Papers of particular interest, published recently, have been highlighted as:

\section{- Of importance}

1. Takahashi K, Yamanaka S. Induction of pluripotent stem cells from mouse embryonic and adult fibroblast cultures by defined factors. Cell. 2006;126(4):663-76.

2. Yu J, Vodyanik MA, Smuga-Otto K, et al. Induced pluripotent stem cell lines derived from human somatic cells. Science. 2007;318(5858):1917-20.

3. Nakajima F, Tokunaga K, Nakatsuji N. Human leukocyte antigen matching estimations in a hypothetical bank of human embryonic stem cell lines in the Japanese population for use in cell transplantation therapy. Stem Cells. 2007;25(4):983-5.

4. Nakatsuji N, Nakajima F, Tokunaga K. HLA-haplotype banking and iPS cells. Nat Biotechnol. 2008;26(7):739-40. 
5. Jia F, Wilson KD, Sun N, et al. A nonviral minicircle vector for deriving human iPS cells. Nat Methods. 2010;7(3):197-9.

6. Huangfu DW, Maehr R, Guo WJ, et al. Induction of pluripotent stem cells by defined factors is greatly improved by small-molecule compounds. Nat Biotechnol. 2008;26(7):795-7.

7. Somers A, Jean JC, Sommer CA, et al. Generation of transgene-free lung disease-specific human induced pluripotent stem cells using a single excisable lentiviral stem cell cassette. Stem Cells. 2010;28(10):1728-40.

8. Sommer CA, Stadtfeld M, Murphy GJ, et al. Induced pluripotent stem cell generation using a single lentiviral stem cell cassette. Stem Cells. 2009;27(3):543-9.

9. Kim D, Kim CH, Moon JI, et al. Generation of human induced pluripotent stem cells by direct delivery of reprogramming proteins. Cell Stem Cell. 2009;4(6):472-6.

10. Miyoshi N, Ishii H, Nagano H, et al. Reprogramming of mouse and human cells to pluripotency using mature MicroRNAs. Cell Stem Cell. $2011 ; 8(6): 633-8$.

11. Anokye-Danso F, Trivedi CM, Juhr D, et al. Highly efficient miRNA-mediated reprogramming of mouse and human somatic cells to pluripotency. Cell Stem Cell. 2011;8(4):376-88.

12. Warren L, Manos PD, Ahfeldt T, et al. Highly efficient reprogramming to pluripotency and directed differentiation of human cells with synthetic modified mRNA. Cell Stem Cell. 2010;7(5):618-30.

13. Cao F, Drukker M, Lin S, et al. Molecular imaging of embryonic stem cell misbehavior and suicide gene ablation. Cloning Stem Cells. 2007;9(1):107-17.

14. Schuldiner M, Itskovitz-Eldor J, Benvenisty N. Selective ablation of human embryonic stem cells expressing a "suicide" gene. Stem Cells. 2003;21(3):257-65.

15. Tang C, Lee AS, Volkmer JP, et al. An antibody against SSEA-5 glycan on human pluripotent stem cells enables removal of teratoma-forming cells. Nat Biotechnol. 2011;29(9):829-34.

16. Carlos Polanco J, Ho MS, Wang B, et al. Identification of unsafe human induced pluripotent stem cell lines using a robust surrogate assay for pluripotency. Stem Cells. 2013;31(8):1498-510.

17. Park IH, Arora N, Huo H, et al. Disease-specific induced pluripotent stem cells. Cell. 2008;134(5):877-86.

18. Pessach IM, Ordovas-Montanes J, Zhang SY, et al. Induced pluripotent stem cells: a novel frontier in the study of human primary immunodeficiencies. J Allergy Clin Immunol. 2011;127(6):1400-7 e4.

19. Wang Y, Jiang Y, Liu S, et al. Generation of induced pluripotent stem cells from human beta-thalassemia fibroblast cells. Cell Res. 2009;19(9):1120-3.

20. Ye L, Chang JC, Lin C, et al. Induced pluripotent stem cells offer new approach to therapy in thalassemia and sickle cell anemia and option in prenatal diagnosis in genetic diseases. Proc Natl Acad Sci U S A. 2009;106(24):9826-30.

21. Itzhaki I, Maizels L, Huber I, et al. Modelling the long QT syndrome with induced pluripotent stem cells. Nature. 2011;471(7337):225-9.

22. Moretti A, Bellin M, Welling A, et al. Patient-specific induced pluripotent stem-cell models for long-QT syndrome. N Engl J Med. 2010;363(15):1397-409.

23. Maehr R, Chen S, Snitow M, et al. Generation of pluripotent stem cells from patients with type 1 diabetes. Proc Natl Acad Sci U S A. 2009;106(37):15768-73.

24. Ebert AD, Yu J, Rose Jr FF, et al. Induced pluripotent stem cells from a spinal muscular atrophy patient. Nature. 2009;457(7227):277-80.

25. Rashid ST, Corbineau S, Hannan N, et al. Modeling inherited metabolic disorders of the liver using human induced pluripotent stem cells. J Clin Invest. 2010;120(9):3127-36.

26. Urbach A, Bar-Nur O, Daley GQ, Benvenisty N. Differential modeling of fragile $\mathrm{X}$ syndrome by human embryonic stem cells and induced pluripotent stem cells. Cell Stem Cell. 2010;6(5):407-11.
27. Marchetto MC, Carromeu C, Acab A, et al. A model for neural development and treatment of Rett syndrome using human induced pluripotent stem cells. Cell. 2010;143(4):527-39.

28. Muotri AR, Marchetto MC, Coufal NG, et al. L1 retrotransposition in neurons is modulated by MeCP2. Nature. 2010;468(7322):443-6.

29. Jiao J, Yang Y, Shi Y, et al. Modeling Dravet syndrome using induced pluripotent stem cells (iPSCs) and directly converted neurons. Hum Mol Genet. 2013;22(21):4241-52.

30. Brennand KJ, Simone A, Jou J, et al. Modelling schizophrenia using human induced pluripotent stem cells. Nature. 2011;473(7346):221-5.

31. Nguyen HN, Byers B, Cord B, et al. LRRK2 mutant iPSC-derived DA neurons demonstrate increased susceptibility to oxidative stress. Cell Stem Cell. 2011;8(3):267-80.

32. Lee G, Papapetrou EP, Kim H, et al. Modelling pathogenesis and treatment of familial dysautonomia using patient-specific iPSCs. Nature. 2009;461(7262):402-6.

33. Lee G, Ramirez CN, Kim H, et al. Large-scale screening using familial dysautonomia induced pluripotent stem cells identifies compounds that rescue IKBKAP expression. Nat Biotechnol. 2012;30(12):1244-8.

34. Hanna J, Wernig M, Markoulaki S, et al. Treatment of sickle cell anemia mouse model with iPS cells generated from autologous skin. Science. 2007;318(5858):1920-3.

35. Raya A, Rodriguez-Piza I, Guenechea G, et al. Disease-corrected haematopoietic progenitors from Fanconi anaemia induced pluripotent stem cells. Nature. 2009;460(7251):53-9.

36. Ardeshirylajimi A, Dinarvand P, Seyedjafari E, et al. Enhanced reconstruction of rat calvarial defects achieved by plasma-treated electrospun scaffolds and induced pluripotent stem cells. Cell Tissue Res. 2013;354(3):849-60.

37. Yan X, Qin H, Qu C, et al. iPS cells reprogrammed from human mesenchymal-like stem/progenitor cells of dental tissue origin. Stem Cells Dev. 2010;19(4):469-80. This publication was the first to demonstrate that iPSC could be generated from cells of dental origin.

38. Miyoshi K, Tsuji D, Kudoh K, et al. Generation of human induced pluripotent stem cells from oral mucosa. J Biosci Bioeng. 2010;110(3):345-50.

39. Oda Y, Yoshimura Y, Ohnishi H, et al. Induction of pluripotent stem cells from human third molar mesenchymal stromal cells. J Biol Chem. 2010;285(38):29270-8.

40. Tamaoki N, Takahashi K, Tanaka T, et al. Dental pulp cells for induced pluripotent stem cell banking. J Dent Res. 2010;89(8):773-8.

41. Wada N, Wang B, Lin NH, et al. Induced pluripotent stem cell lines derived from human gingival fibroblasts and periodontal ligament fibroblasts. J Periodontal Res 2011;46(4):438-47.

42. Nomura Y, Ishikawa M, Yashiro Y, et al. Human periodontal ligament fibroblasts are the optimal cell source for induced pluripotent stem cells. Histochem Cell Biol. 2012;137(6):719-32.

43. Egusa $\mathrm{H}$, Okita $\mathrm{K}$, Kayashima $\mathrm{H}$, et al. Gingival fibroblasts as a promising source of induced pluripotent stem cells. PLoS One. 2010;5(9):e12743.

44. Beltrao-Braga PI, Pignatari GC, Maiorka PC, et al. Feeder-free derivation of induced pluripotent stem cells from human immature dental pulp stem cells. Cell Transplant. 2011.

45. Kim K, Doi A, Wen B, et al. Epigenetic memory in induced pluripotent stem cells. Nature. 2010;467(7313):285-90.

46. Polo JM, Liu S, Figueroa ME, et al. Cell type of origin influences the molecular and functional properties of mouse induced pluripotent stem cells. Nat Biotechnol. 2010;28(8):848-55.

47. Volponi AA, Pang Y, Sharpe PT. Stem cell-based biological tooth repair and regeneration. Trends Cell Biol. 2010;20(12):715-22.

48. Arakaki M, Ishikawa M, Nakamura T, et al. Role of epithelial-stem cell interactions during dental cell differentiation. J Biol Chem. 2012;287(13):10590-601. This publication was the first to demonstrate that mouse iPSC have the ability to differentiate into dental tissue. 
49. Otsu K, Kishigami R, Oikawa-Sasaki A, et al. Differentiation of induced pluripotent stem cells into dental mesenchymal cells. Stem Cells Dev. 2012;21(7):1156-64.

50. Chai Y, Jiang X, Ito Y, et al. Fate of the mammalian cranial neural crest during tooth and mandibular morphogenesis. Development. 2000;127(8):1671-9.

51. Pispa J, Thesleff I. Mechanisms of ectodermal organogenesis. Dev Biol. 2003;262(2):195-205.

52. Imai H, Osumi-Yamashita N, Ninomiya Y, Eto K. Contribution of early-emigrating midbrain crest cells to the dental mesenchyme of mandibular molar teeth in rat embryos. Dev Biol. 1996; 176(2):151-65.

53. Wen Y, Wang F, Zhang W, et al. Application of induced pluripotent stem cells in generation of a tissue-engineered tooth-like structure. Tissue Eng Part A. 2012;18(15-16): 1677-85.

54. Cai J, Zhang Y, Liu P, et al. Generation of tooth-like structures from intergration-free human urine induced pluripotent stem cells. Cell Regen. 2013;2:6. This publication was the first to demonstrate that human iPSC are capable of contributing to the formation of toothlike structures.

55. Duan X, Tu Q, Zhang J, et al. Application of induced pluripotent stem (iPS) cells in periodontal tissue regeneration. J Cell Physiol. 2011;226(1):150-7.

56. Heijl L, Heden G, Svardstrom G, Ostgren A. Enamel matrix derivative (EMDOGAIN) in the treatment of intrabony periodontal defects. J Clin Periodontol. 1997;24(9 Pt 2):705-14.

57. Hirooka $\mathrm{H}$. The biologic concept for the use of enamel matrix protein: true periodontal regeneration. Quintessence Int. 1998;29 (10):621-30.
58. Keila S, Nemcovsky CE, Moses O, et al. In vitro effects of enamel matrix proteins on rat bone marrow cells and gingival fibroblasts. $\mathrm{J}$ Dent Res. 2004;83(2):134-8.

59. Lyngstadaas SP, Wohlfahrt JC, Brookes SJ, et al. Enamel matrix proteins; old molecules for new applications. Orthod Craniofac Res. 2009;12(3):243-53.

60. Sculean A, Nikolidakis D, Schwarz F. Regeneration of periodontal tissues: combinations of barrier membranes and grafting materials biological foundation and preclinical evidence: a systematic review. J Clin Periodontol. 2008;35(8 Suppl):106-16.

61. Hynes K, Menicanin D, Han J, et al. Mesenchymal stem cells from iPS cells facilitate periodontal regeneration. J Dent Res. 2013;92(9): 833-9. This publication was the first to demonstrate that MSC-like cells derived from iPSC are capable of enhancing periodontal tissue regeneration.

62. Lian Q, Zhang Y, Zhang J, et al. Functional mesenchymal stem cells derived from human induced pluripotent stem cells attenuate limb ischemia in mice. Circulation. 2010;121(9):1113-23.

63. Karlsson C, Emanuelsson K, Wessberg F, et al. Human embryonic stem cell-derived mesenchymal progenitors-Potential in regenerative medicine. Stem Cell Res. 2009;3(1):39-50.

64. Kuo ML. Efficient derivation \& concise gene expression profiling of human embryonic stem cell-derived mesenchymal progenitors (EMPs). Cell Transplant. 2011;20(10):1529-45.

65. Villa-Diaz LG, Brown SE, Liu Y, et al. Derivation of mesenchymal stem cells from human induced pluripotent stem cells cultured on synthetic substrates. Stem Cells. 2012;30(6):1174-81.

66. Wei H, Tan G, Manasi, et al. One-step derivation of cardiomyocytes and mesenchymal stem cells from human pluripotent stem cells. Stem Cell Res. 2012;9(2):87-100. 